\title{
A Study of the Suitability of IrOBEX for High-Speed Exchange of Large Data Objects
}

\author{
Casey T. Deccio \\ casey@byu.net \\ Joseph Ekstrom \\ Charles D. Knutson \\ knutson@cs.byu.edu \\ D. Ryan Partridge \\ Kevin B. Tew
}

Follow this and additional works at: https://scholarsarchive.byu.edu/facpub

Part of the Computer Sciences Commons

\section{Original Publication Citation}

Casey T. Deccio, Joseph Ekstrom, D. Ryan Partridge, Kevin B. Tew, and Charles D. Knutson. "A Study of the Suitability of IrOBEX for High-Speed Exchange of Large Data Objects." Proceedings of the IEEE Global Communications Conference 23.

\section{BYU ScholarsArchive Citation}

Deccio, Casey T.; Ekstrom, Joseph; Knutson, Charles D.; Partridge, D. Ryan; and Tew, Kevin B., "A Study of the Suitability of IrOBEX for High-Speed Exchange of Large Data Objects" (2003). Faculty Publications. 1051.

https://scholarsarchive.byu.edu/facpub/1051

This Peer-Reviewed Article is brought to you for free and open access by BYU ScholarsArchive. It has been accepted for inclusion in Faculty Publications by an authorized administrator of BYU ScholarsArchive. For more information, please contact ellen_amatangelo@byu.edu. 


\title{
A Study of the Suitability of IrOBEX for High-Speed Exchange of Large Data Objects
}

\author{
Casey T. Deccio, Joseph Ekstrom, D. Ryan Partridge, Kevin B. Tew, and Charles D. Knutson \\ \{cdeccio, jce, rpartrid, tewk, knutson\}@ cs.byu.edu
}

\begin{abstract}
This paper demonstrates that careful tuning of the OBEX and IrLAP negotiated parameters allows OBEX to scale well for use with large data objects and high transmission rates. Due to the substantial time overhead inherent in link turnarounds, minimizing turnarounds during the transmission of a large object helps to maximimize link efficiency. The IrLAP window size and OBEX packet size significantly impact the number of required turnarounds during the transmission of a large object. When these parameters are properly tuned, maximum throughput can be achieved, and OBEX performs efficiently at high data rates.
\end{abstract}

Index Terms-Object Exchange, OBEX, IrDA, IrLAP

\section{INTRODUCTION}

O BJECT EXCHANGE (OBEX) [1] is a binary session layer protocol designed to transmit data objects between resource-limited devices. It is based on HTTP, which has become a worldwide standard for object exchange across the Internet. Since it is binary rather than text-based, OBEX is an attractive option for resource-constrained wireless devices because processing overhead is much lower than for traditional text-based protocols. OBEX was originally developed by the Infrared Data Association (IrDA) for infrared wireless devices, and has since been adopted by the Bluetooth SIG as a standard for object exchange.

The wireless technology market has experienced tremendous growth. Users today expect to be able to communicate and access data anytime, anywhere, using almost any device. Traditional mobile devices such as cell phones and PDAs must now handle complex jobs involving large files, such as MP3 audio and MPEG video. The demand for efficient transmission of large data and media objects between wireless devices at increasingly higher speeds calls into question the scalability of the OBEX protocol.

In [2] it was shown that the IrDA Link Access Protocol (IrLAP) [3] scales to a data rate of $100 \mathrm{Mbps}$ without modification. Building on that work, this paper shows that careful tuning of the OBEX and IrLAP negotiated parameters allows OBEX to scale well for use with large data objects and high transmission rates.

The rest of the paper is organized as follows: In section II we discuss the IrLAP and OBEX protocols and their key parameters. In section III we introduce the mathematical model on which we have based our study. Section IV presents our experimental results. In Section $\mathrm{V}$ we present our conclusions.

\section{BACKGROUND}

In order to properly tune OBEX for maximum performance, it is necessary to understand the underlying link layer. IrLMP

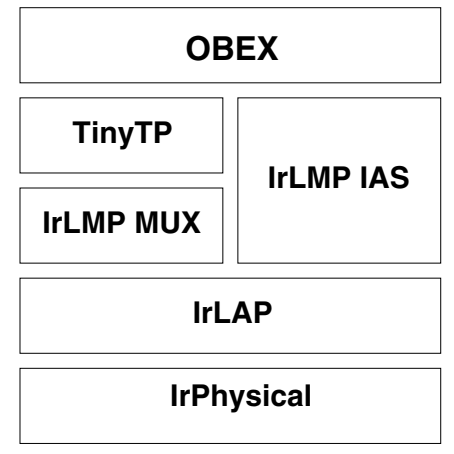

Figure 1. OBEX and the IrDA protocol stack. [1] [3] [4] [5].

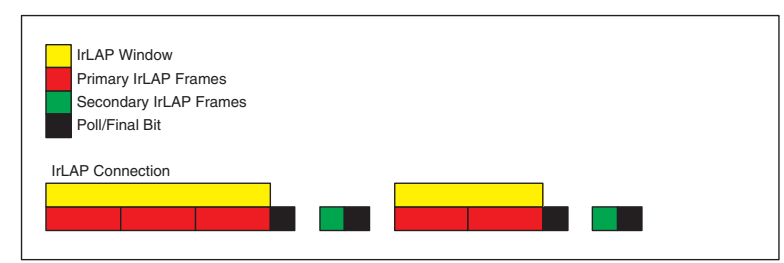

Figure 2. Example IrLAP windows.

[4] and TinyTP [5] add a constant 3 byte overhead but do not influence the IrLap or OBEX parameters in any other way. This section is a brief overview of the IrLAP and OBEX protocols, and highlights key parameters that impact performance.

\section{A. IrLAP}

The Link Access Protocol (IrLAP) layer [3] establishes and manages the link between a primary and a secondary infrared device (see Figure 1). Because point-to-point infrared communication is inherently half-duplex, the receiving device may not transmit until it receives a "clear to send" from the transmitting device. A "clear to send" is signaled when the poll/final bit of an IrLAP frame is set, forcing a link turnaround. A device

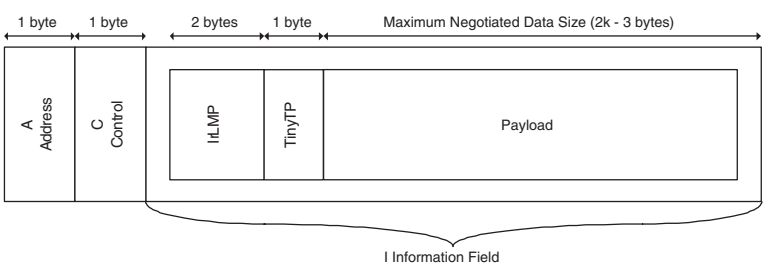

Figure 3. IrLAP Frame. 
may send a window of multiple unacknowledged frames before setting the poll/final bit (see Figure 2).

Several parameters are negotiated when a link is established. Window sizes and minimum turn around time in the VFIR specification are 127 and $100 \mathrm{~ms}$ respectively [6]. In [2] the authors base their research parameters on the VFIR specification, but make allowances for the faster $100 \mathrm{Mbit}$ transmission speed. It should be noted that 100 Mbit IrDA is purely theoretical and has no standard specification. Of these parameters, minimum turnaround time, frame length, and window size directly impact link performance. The minimum turnaround time is the latency required by a transmitting device after it sends a packet with the poll/final bit set, and before the other device is permitted to begin transmitting. Some factors that determine the minimum turnaround time are photo receptor energy dissipation time, device interrupt latency, data processing overhead, and memory storage latency. Frame length is the maximum size of the Information field in an IrLAP frame (see Figure 3) [3]. Window size is the maximum number of unacknowledged frames that can be sent before the link must be turned around. Frame length and window size dictate the frequency with which a link must be turned around, and hence are critical to link utilization efficiency. Table I illustrates the impact of these two parameters on aggregate overhead due to link turnaround during transmission of a single (relatively small) data object. In this table, object size is assumed to be $10 \mathrm{MB}$ and turnaround time is assumed to be $2 \mathrm{~ms}$.

TABLE I

IMPACT OF FRAME LENGTH AND WINDOW SIZE ON IRLAP TURNAROUNDS

\begin{tabular}{|c|c|c|c|}
\hline $\begin{array}{c}\text { Frame } \\
\text { Length }\end{array}$ & $\begin{array}{c}\text { Window } \\
\text { Size }\end{array}$ & $\begin{array}{c}\text { Number of } \\
\text { Turnarounds }\end{array}$ & $\begin{array}{c}\text { Total } \\
\text { Turnaround Time }\end{array}$ \\
\hline 512 B & 1 & 20480 & $40.96 \mathrm{~s}$ \\
\hline $2 \mathrm{~KB}$ & 1 & 5120 & $10.24 \mathrm{~s}$ \\
\hline $512 \mathrm{~B}$ & 7 & 2926 & $5.85 \mathrm{~s}$ \\
\hline $2 \mathrm{~KB}$ & 7 & 731 & $1.46 \mathrm{~s}$ \\
\hline $512 \mathrm{~B}$ & 127 & 161 & $322 \mathrm{~ms}$ \\
\hline $2 \mathrm{~KB}$ & 127 & 40 & $80 \mathrm{~ms}$ \\
\hline
\end{tabular}

\section{B. $O B E X$}

All information carried by OBEX is encapsulated in OBEX headers, conceptually similar to headers in HTTP. These headers may contain meta-data about the object (such as name, length, MIME-type, etc.) and may include one or more body headers containing the object itself. Each header is either fixed length or length prefixed [1].

OBEX transmits objects using GET and PUT methods similar to HTTP. The OBEX 1.3 specification defines a 2 byte packet length header field resulting in a maximum OBEX packet length of $64 \mathrm{~KB}$ [1]. This packet size limitation requires that GET and PUT operations for large objects be segmented into multiple OBEX packets. The OBEX standard requires that each request packet transmitted in either a GET or PUT operation must be acknowledged by a CONTINUE response packet, resulting in an effective session layer window size of one [1].

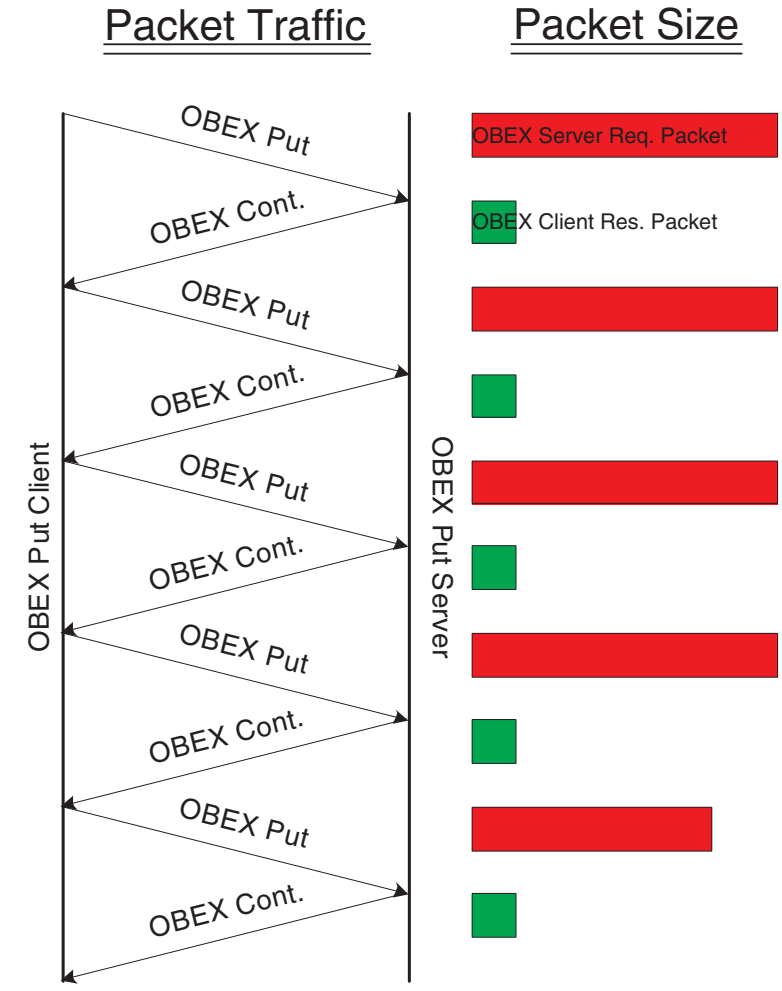

Figure 4. OBEX Conversation.

Because of the half duplex nature of IrDA and the effective OBEX window size of one, subsequent request packets cannot be transmitted until a CONTINUE acknowledgment is received (see Figure 4). Therefore, each request/response pair generates two turnarounds, one after the request, and a second after the CONTINUE response.

\section{IRDA AND OBEX MODEL}

This section presents a mathematical model of the interactions and behavior of OBEX and IrLAP. Variables presented in this section are defined in Table II. The first equation models the number of OBEX layer turnarounds. We build on this equation to derive a model for the number of turnarounds at the IrLAP layer. Finally, based on these two equations, we construct a model for the time necessary to transmit an object of arbitrary size using OBEX over IrLAP.

\section{A. OBEX Turnarounds}

Figure 5 illustrates the way in which OBEX packetizes a large object for transmission. The first packet in an OBEX PUT operation typically contains object meta-information (such as name, length, etc.), although it is not strictly required to do so. A body header may or may not be included in this first packet, but if included is normally shorter than in subsequent packets, due to the space occupied by headers for meta-information. While no specific requirements are imposed on the order or content of the first packet, for the sake of our model we assume that the first packet includes name and length headers plus a necessarily shortened body header. The value 10 in the following 


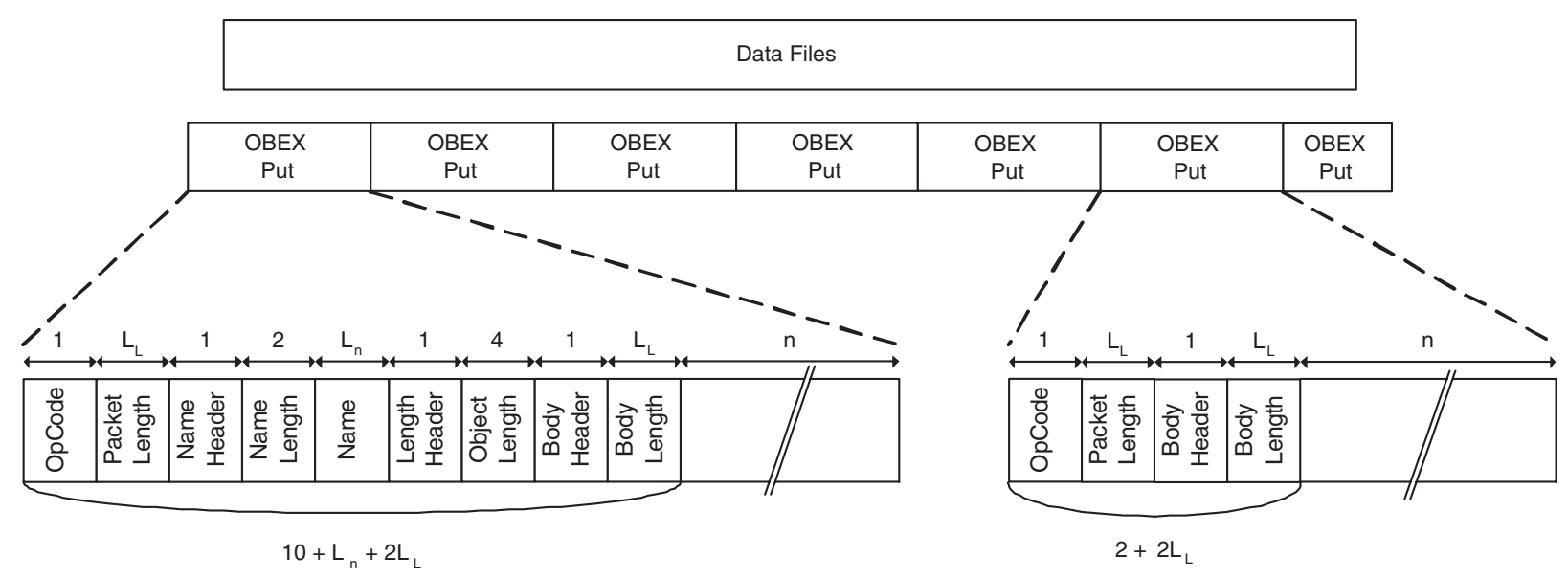

Figure 5. OBEX object packetization. The first packet has additional headers that specify object length and object name. Subsequent packet headers only need to contain the packet and body lengths.

TABLE II

Mathematical Model Variables

\begin{tabular}{|c|c|}
\hline$T_{O}$ & number of OBEX turnarounds \\
\hline$T_{L}$ & number of IrLAP turnarounds \\
\hline$s_{O}$ & object size (bytes) \\
\hline$p_{0}$ & payload size of first OBEX packet (bytes) \\
\hline$p_{n}$ & payload size of all \\
& subsequent OBEX packets (bytes) \\
\hline$s_{p}$ & OBEX maximum packet size (bytes) \\
\hline$L_{N}$ & length of OBEX name field (bytes) \\
\hline$L_{L}$ & length of OBEX length field (bytes) \\
\hline$B_{r e m}$ & $\begin{array}{c}\text { bytes that account for an } \\
\text { incomplete OBEX packet (bytes) }\end{array}$ \\
\hline$f$ & IrLAP frame size (bytes) \\
\hline$i$ & payload size of I field (bytes) \\
\hline$w$ & IrLAP window size \\
\hline$r$ & transmission rate (bits/sec) \\
\hline$t$ & transmission time (sec) \\
\hline$t_{s}$ & time to send data to receiver \\
\hline$t_{w}$ & $\begin{array}{r}\text { latency imposed by min turnaround time } \\
\text { and processing overhead }\end{array}$ \\
\hline$t_{r}$ & time for receiver to transmit a response \\
\hline
\end{tabular}

equation reflects the constant overhead of the name and length headers. $L_{N}$ and $2 L_{L}$ reflect the variable length overhead associated with these headers.

$$
p_{0}=s_{p}-\left(10+L_{N}+2 L_{L}\right)
$$

For subsequent packets, we assume a common case in which packets consist entirely of body headers, incurring only the overhead of the packet length field and the body header length field.

$$
p_{n}=s_{p}-\left(2+2 L_{L}\right)
$$

Although the OBEX specification fixes the length field at two bytes, we have chosen to make it a variable in order to allow for experimentation with packet sizes greater than $64 \mathrm{~KB}$.
Since the first OBEX packet has a different payload capacity than subsequent packets, we subtract $p_{0}$ from $s_{o}$ and then divide by $p_{n}$ to yield the number of packets with payload size $p_{n}$ required to transmit the object. To this, we add one to account for the first packet (with payload size $p_{0}$ ). Because each OBEX request packet must be acknowledged with a $\mathrm{CON}$ TINUE response packet, the number of packets sent by the sender and receiver is symmetrical, therefore we multiply by two. Since each OBEX packet requires a turnaround, the number of OBEX packets is analogous to the number of required OBEX turnarounds.

This yields the following equation, which models OBEX turnarounds as a function of object size.

$$
T_{O}=2\left(\left\lceil\frac{s_{o}-p_{0}}{p_{n}}\right\rceil+1\right)
$$

\section{B. IrLAP Turnarounds}

IrLAP turnarounds are dependent upon IrLAP windows, which are calculated with respect to OBEX packets. The number of full OBEX packets, or packets of size $s_{p}$, is denoted by $\left\lfloor\frac{s_{o}-p_{o}}{p_{n}}+1\right\rfloor$. The final OBEX packet will normally be less than full, and has size $B_{r e m}$ which is given by

$$
\begin{aligned}
B_{\text {rem }}=\left(\left(s_{o}-p_{0}\right) \bmod p_{n}\right) \\
+\left(\left\lceil\frac{\left(s_{o}-p_{0}\right) \bmod p_{n}}{p_{n}}\right\rceil\right) *\left(2+2 L_{L}\right)
\end{aligned}
$$

Each full OBEX packet is transmitted in $\left\lceil\frac{s_{p}}{i * w}\right\rceil$ IrLAP windows. Similarly, $B_{\text {rem }}$ requires $\left\lceil\frac{B_{r e m}}{i * w}\right\rceil$ IrLAP windows for transmission. We multiply the number of IrLAP windows for a single OBEX packet by the number of OBEX packets, and add the number of IrLAP windows for the under-filled packet to produce the total number of windows required to transmit an object of size $s_{o}$. Each IrLAP window requires an acknowledgment, so the number of windows is identical to the number of turnarounds, and hence must be multiplied by two. 


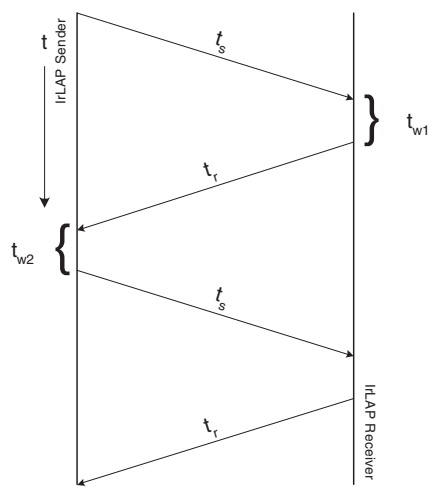

Figure 6. IrLAP transmission time diagram. $t_{s}$ is sender's transmission time. $t_{r}$ is receivers transmission time. $t_{w 1}$ and $t_{w 2}$ are the turnaround time for the receiver and sender respectively

The resulting equation for IrLAP turnarounds is

$$
T_{L}=2\left(\left(\left\lceil\frac{s_{p}}{i * w}\right\rceil *\left\lfloor\frac{s_{o}-p_{0}}{p_{n}}+1\right\rfloor\right)+\left\lceil\frac{B_{r e m}}{i * w}\right\rceil\right)
$$

\section{Transmission Time}

The general formula for the time required to transmit data, receive a response, and be ready to transmit again is given by

$$
t_{\text {round-trip }}=t_{s}+t_{w 1}+t_{r}+t_{w 2}
$$

A single IrDA round trip is illustrated by Figure 6 . We have defined $t_{s}=\frac{(f+2) * w}{r}$, where 2 is the size of the Address and Control fields of the IrLAP frame (see Figure 3). The time equation is divided into three smaller equations, shown below. $t_{\text {full }}$ is the time required to transmit full OBEX packets. $t_{\text {partial }}$ represents the time for an under-filled OBEX packet that is larger than one IrLAP window. $t_{\text {end }}$ is the time due to any remaining bytes that do not completely fill one IrLAP window.

$$
\begin{gathered}
t_{\text {full }}=t_{\text {round-trip }} *\left(\left\lceil\frac{s_{p}}{i * w}\right\rceil *\left\lfloor\frac{s_{o}-p_{0}}{p_{n}}+1\right\rfloor\right) \\
t_{\text {partial }}=t_{\text {round-trip }} *\left(\left\lfloor\frac{B_{\text {rem }}}{i * w}\right\rfloor\right) \\
t_{\text {end }}=\frac{B_{\text {rem }} \bmod (i * w)}{r}+t_{w 1}+t_{r}+t_{w 2}
\end{gathered}
$$

Equations (7) and (8) are derived directly from the two terms in (5). They represent the time required to send one complete IrLAP window multiplied by the number of IrLAP windows represented by each term. The last equation, (9) is the time to transmit any remaining bytes smaller than one IrLAP window in size. Summing (7), (8), and (9) yields the total amount of time (in seconds) required to transmit an OBEX object of a given size.

$$
t=t_{\text {full }}+t_{\text {partial }}+t_{\text {end }}
$$

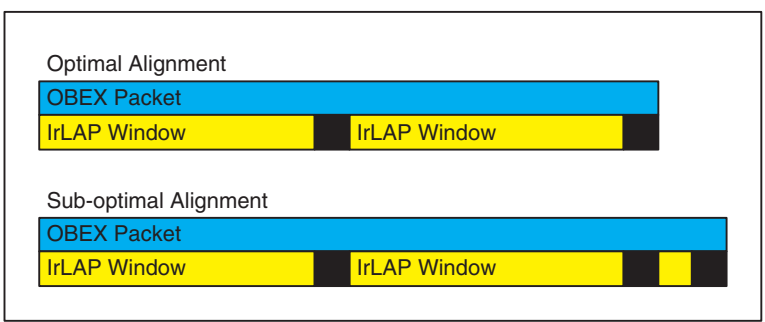

Figure 7. OBEX packet alignment with IrLAP windows.

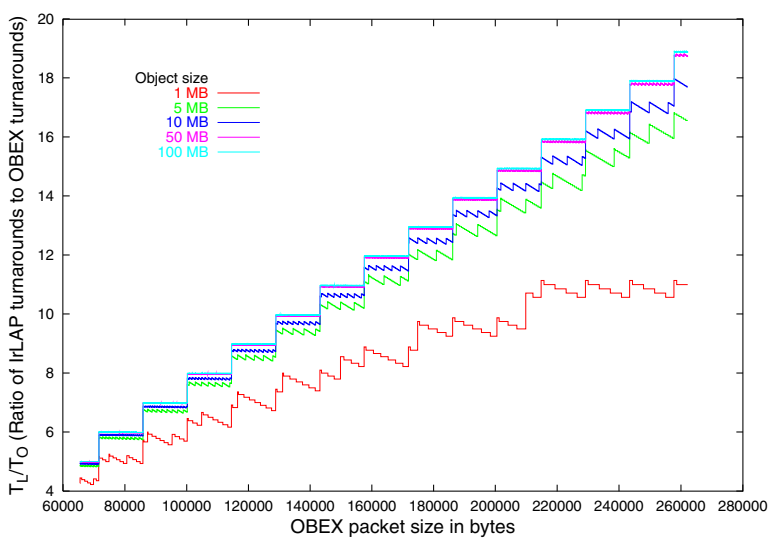

Figure 8. Ratio of IrLAP turnarounds $\left(T_{L}\right)$ to OBEX turnarounds $\left(T_{O}\right)$ for OBEX packet sizes of $64 \mathrm{~KB}$ to $256 \mathrm{~KB}$, showing five object sizes $(1,5,10$, 50 , and $100 \mathrm{MB}$ ).

\section{RESULTS}

\section{A. OBEX - IrLAP Turnaround Ratio}

In this section we apply our model to a number of scenarios in order to characterize the performance of OBEX as data objects increase in size and as transmission speeds increase. Our first experiment compares the ratio of IrLAP turnarounds to OBEX turnarounds as OBEX maximum packet size increases beyond the specified $64 \mathrm{~KB}$ limit. For this experiment, the IrLAP frame size is $2 \mathrm{~KB}$ and the window size is 7. As OBEX packet size increases, the number of OBEX turnarounds will obviously decrease. However, of particular interest is the effect that larger OBEX packet sizes have on the IrLAP/OBEX turnaround ratio.

Figure 7 illustrates the manner in which OBEX packets are segmented into underlying IrLAP windows. As OBEX maximum packet size increases, it approaches optimal IrLAP window utilization. Immediately beyond this point, each OBEX packet requires one more nearly empty IrLAP window. Figure 8 shows the effect of IrLAP window utilization on turnaround ratio. Note the series of gradual ratio decreases, each followed by a sharp increase. The local minima, or "sweet spots", occur when OBEX packet size achieves optimal alignment with IrLAP window boundaries. The significance of these "sweet spots" is illustrated when considering total object transmission time.

\section{B. Object Transmission Time}

This section explores the effect of varying the IrLAP window size while holding the OBEX packet size constant. This 


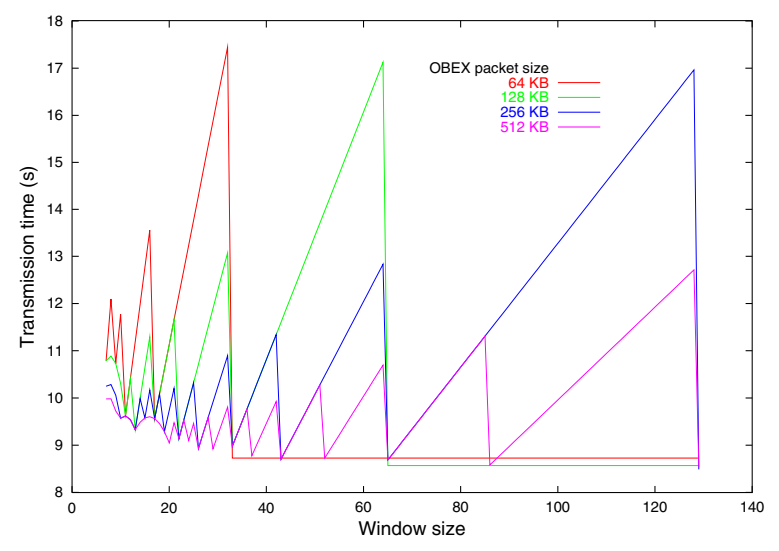

Figure 9. Transmission time of a $100 \mathrm{MB}$ object for increasing IrLAP window sizes, showing OBEX packet sizes from $64 \mathrm{~KB}$ to $512 \mathrm{~KB}$.

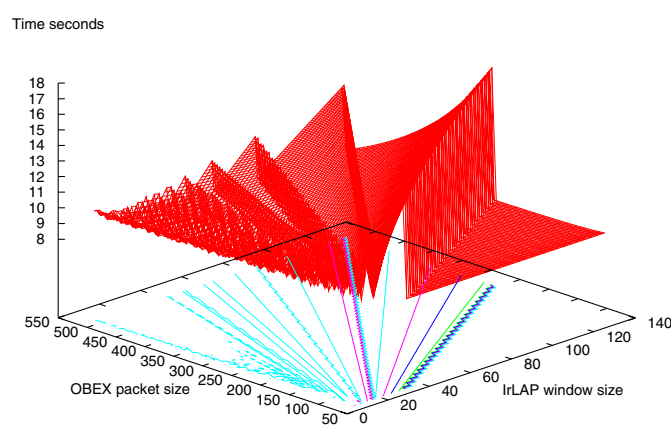

Figure 10. Relationship between window size and packet size.

experiment models the transmission of a $100 \mathrm{MB}$ object where $i=2 \mathrm{~KB}, r=100 \mathrm{Mbps}$, and $w$ varies from 7 to 127 . Figure 9 shows that transmission time reaches a (local) maximum for certain IrLAP window sizes, dropping immediately thereafter to a (local) minimum. These local minima and maxima reflect the effect of the "sweet spots" on transmission time. In other words, transmission time is smallest when OBEX packet size falls near IrLAP window boundaries, avoiding unnecessary additional IrLAP turnarounds. Table III summarizes the minimum transmission times for OBEX packet sizes 64, 128, 256, and $512 \mathrm{~KB}$.

TABLE III

Transmission Time for Various ObEX Packet Sizes

\begin{tabular}{|c|c|c|c|}
\hline $\begin{array}{c}\text { OBEX } \\
\text { Packet Size }\end{array}$ & $\begin{array}{c}\text { Optimal } \\
\text { Window Size }\end{array}$ & $\begin{array}{c}\text { Minimum } \\
\text { Trans. Time }\end{array}$ & $\begin{array}{c}\text { Link } \\
\text { Utilization }\end{array}$ \\
\hline $64 \mathrm{~KB}$ & 33 & $8.73 \mathrm{~s}$ & $95.99 \%$ \\
\hline $128 \mathrm{~KB}$ & 65 & $8.57 \mathrm{~s}$ & $97.83 \%$ \\
\hline $256 \mathrm{~KB}$ & 129 & $8.49 \mathrm{~s}$ & $98.70 \%$ \\
\hline $512 \mathrm{~KB}$ & 257 & $8.40 \mathrm{~s}$ & $99.76 \%$ \\
\hline \multicolumn{2}{|c|}{ Theoretical Min. Time } & $8.38 \mathrm{~s}$ & \\
\hline
\end{tabular}

$s_{o}=100 \mathrm{MB}, i=2 \mathrm{~KB}$

Figure 10 illustrates the performance surface formed by
OBEX packet size and IrLAP window size parameters. The flat plateau region to the right is where IrLAP window size has met or exceeded the OBEX packet size. The valleys correspond to "sweet spots" as previously described. The ridges depict the worst case combinations of maximum OBEX packet size and IrLAP window size which may reduce throughput by as much as $50 \%$.

\section{CONCLUSION}

We have shown that the OBEX protocol, when tuned to maximize IrLAP performance, scales to handle high data rates and large objects. While OBEX packet / IrLAP window alignment is the key to OBEX performance tuning, perfect alignment is not necessary. Slightly under-filled IrLAP windows achieve near optimal data throughput. However, slightly overfilled IrLAP frames produce a significantly detrimental effect on throughput, decreasing performance by nearly $50 \%$. Packet sizes that are powers of two (ironically the default specifications for maximum OBEX packet sizes and IrLAP window sizes) result in the worst possible performance due to slightly overfilled IrLAP windows. These negative effects can be mitigated by appropriate tuning of packet sizes within the OBEX session layer.

\section{REFERENCES}

[1] "Irda object exchange protocol obex," Infrared Data Association, Walnut Creek, Calfornia, 2003.

[2] A. C. Boucouvalas and V. Vitsas, "100 mb/s irda protocol performance evaluation," in Proceedings of IASTED International Conference on Wireless and Optical Communications (WOC 2001), Banff, Canada, June 2001, pp. 49-57.

[3] "Serial infrared link access protocol (irlap)," Infrared Data Association, Walnut Creek, Calfornia, 1996.

[4] "Link management protocol," Infrared Data Association, Walnut Creek, Calfornia, 1996.

[5] "Tiny tp: A flow-control mechanism for use with irlmp," Infrared Data Association, Walnut Creek, Calfornia, 1996.

[6] "Serial infrared physical layer specification for $16 \mathrm{mb} / \mathrm{s}$ addition (vfir)," Infrared Data Association, Walnut Creek, Calfornia, 1999.

[7] "Serial infrared physical layer specification (irphy)," Infrared Data Association, Walnut Creek, Calfornia, 1998 